\title{
Cryogenic permanent magnet undulators
}

\author{
Toru Hara, ${ }^{*}$ Takashi Tanaka, and Hideo Kitamura \\ RIKEN/SPring-8, 1-1-1 Kouto, Mikazuki-cho, Sayo-gun, Hyogo 679-5148, Japan \\ Teruhiko Bizen, Xavier Maréchal, and Takamitsu Seike \\ JASRI/SPring-8, 1-1-1 Kouto, Mikazuki-cho, Sayo-gun, Hyogo 679-5198, Japan
}

Tsutomu Kohda and Yutaka Matsuura

Sumitomo Special Metal Co., Ltd., 2-15-17 Egawa, Shimamoto-cho, Mishima-gun, Osaka 618-0013, Japan

(Received 7 January 2004; published 18 May 2004)

\begin{abstract}
In order to obtain high magnetic fields in a short period undulator, superconductive undulators have been actively investigated in recent years. In this paper, however, we propose a new approach, the cryogenic permanent magnet undulator (CPMU) design, using permanent magnets at the cryogenic temperature of liquid nitrogen or higher. This cryogenic scheme can be easily adapted to currently existing in-vacuum undulators and it improves the magnetic field performance by $30 \%-50 \%$. Unlike superconductive undulators operating around the liquid helium temperature, there is no big technological difficulty such as the thermal budget problem. In addition, existing field correction techniques are applicable to the CPMUs. Since there is no quench in the CPMUs, the operation of the CPMUs has the same reliability as conventional permanent magnet undulators.
\end{abstract}

DOI: 10.1103/PhysRevSTAB.7.050702

PACS numbers: 07.85.Qe, 41.60.Ap, 07.55.Db

\section{INTRODUCTION}

In recent years, short period undulators became widely used in synchrotron radiation facilities, because they increase the number of periods in a unit undulator length and as a consequence, they generate brighter synchrotron radiation. Moreover, a short undulator periodicity enables the emission of high energy photons, and therefore it opens the way for an $\mathrm{x}$-ray beam line operation in medium size synchrotron radiation facilities, such as SLS and NSLS [1,2]. For the same reason, a short period undulator is very attractive for SASE-FEL facilities, since it lowers the electron beam energy necessary for an $\mathrm{x}$-ray FEL operation. This makes the whole facility design compact and economical [3].

In general, when reducing the undulator periodic length, the dimensions of each magnet piece become smaller, and consequently the undulator should be operated at smaller magnetic gaps in order to obtain sufficient magnetic fields. One big advance for the realization of short period undulators has been the in-vacuum design, which accommodates permanent magnet arrays inside vacuum and eliminates the physical limitation of the magnetic gap due to the vacuum chamber [4]. In-vacuum undulators have been successfully operated for years in many synchrotron radiation facilities $[1,2,4,5]$.

Another prospective technology is superconductive magnets. For its high magnetic field, the development of superconductive undulators (SCUs) has been continued

*Electronic address: toru@spring8.or.jp for more than ten years [6], and a 1.3 T SCU was recently developed with a $14 \mathrm{~mm}$ period at a gap of $5 \mathrm{~mm}$ [7-9]. Although the performance of wire materials and cryogenics has been steadily improved, SCUs should be operated around the temperature of liquid helium and there still remains the thermal budget problem. There are two options for the cryogenic design of SCUs, with or without a thermal shield between the superconductive magnets and the electron beams. Since most facilities do not have helium liquefiers installed on site, SCUs should be normally operated with cryocoolers having a cooling capacity of a few watts at the temperature of liquid helium. Therefore in the case of the no thermal shield design, serious consideration is needed regarding the heat produced by the resistive wall effect and synchrotron radiation coming from upstream bending magnets in a storage ring $[8,10]$. In order to avoid a temperature rise and quench, a thermal shield can be inserted, but it enlarges the magnetic gap and results in considerable deterioration of the field performance. Another concern over SCUs is a procedure of magnetic field correction, particularly for the phase error, which is an important parameter for undulators as well as the integrated multipole components of the magnetic fields. Since conventional field correction schemes used for permanent magnet undulators cannot be directly applied to superconductive devices, the establishment of new field correction methods is indispensable $[6,8]$.

In this paper, a new approach for the construction of a high field short period undulator is proposed. As is well known, NdFeB magnets exhibit an increase of their remanent fields and coercivity as lowering the temperature [11]. By exploiting this nature in the permanent magnet 
undulator technology, the performance of the magnetic field can be drastically improved.

\section{CRYOGENIC PERMANENT MAGNET UNDULATORS}

$\mathrm{NdFeB}$ magnets with high coercivity or $\mathrm{Sm}_{2} \mathrm{Co}_{17}$ magnets are generally used in in-vacuum undulators because of their resistance against demagnetization due to electron beam irradiation. In addition, when installed in a storage ring, thermal stabilization and vacuum bakeout at high temperatures around $420 \mathrm{~K}$ are necessary for the magnets; high coercivity magnets are also favored to minimize thermal demagnetization during these processes [12]. However, if the undulator is operated at a cryogenic temperature, the outgassing rate from the magnets becomes very low or the magnets are even expected to work as cryopumps, so that it is no more necessary to expose the magnets to high temperatures. Supposing a cryogenic temperature operation, $\mathrm{NdFeB}$ magnets with high remanent fields, normally showing low coercivity at room temperature, are expected to have sufficiently high coercivity and resistivity against electron beam irradiation. This gives us an opportunity to create a new undulator concept called the cryogenic permanent magnet undulators (CPMUs).

Since the magnet arrays of an in-vacuum undulator are placed under good thermal isolation with vacuum, the undulator operation at the cryogenic temperature of liquid nitrogen or higher simply needs some additional refrigerant channels or cryocoolers. Figure 1 shows two

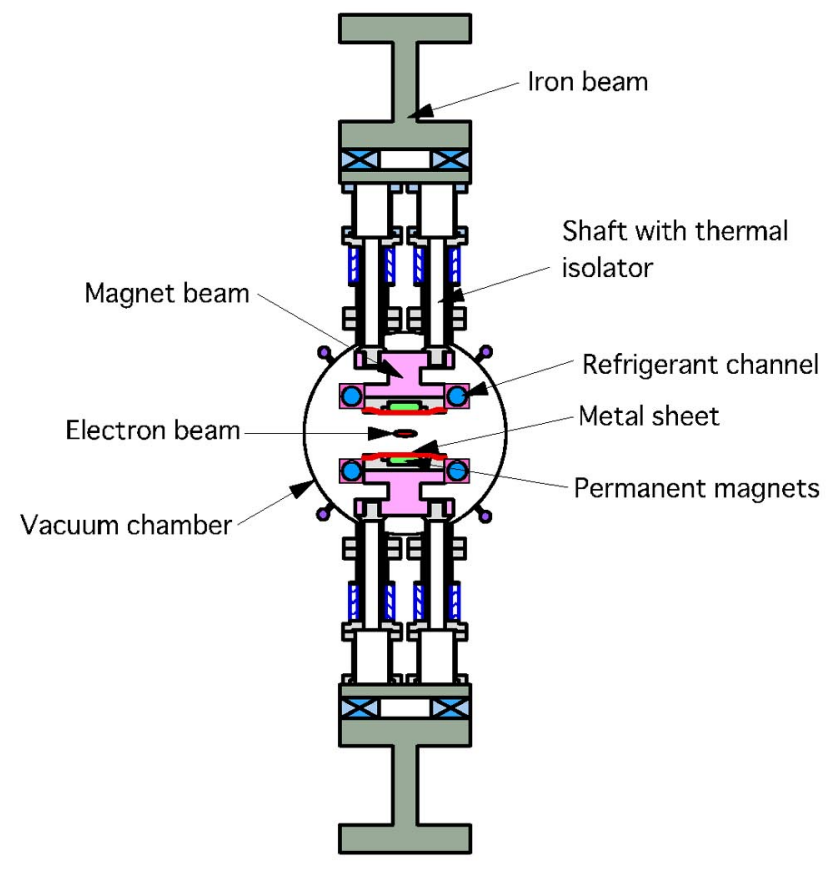

(a) examples of the CPMU design, both of which resemble the ordinary in-vacuum undulator design [12] except having refrigerant channels 1(a) or cryocoolers 1(b) attached to the magnet beams.

The most important advantage of the CPMUs is to allow a very high heat load of several hundred watts, which can be covered by a compact cryocooler of a Gifford McMahon type. In case of a $1.5 \mathrm{~m}$ CPMU, the estimated amount of heat flowing in through the shafts of the magnet beams is about $100 \mathrm{~W}$ and thermal radiation from the inner surface of the vacuum chamber is about $15 \mathrm{~W}$. The heat generated by the resistive wall effect and synchrotron radiation from upstream bending magnets is normally smaller, for instance about $10 \mathrm{~W}$ in the case of the 203-bunch operation in SPring- 8 at a $3 \mathrm{~mm}$ gap. These heat loads can be covered by one cryocooler, for example, the Suzuki Shokan RF90S having a cooling capacity higher than $200 \mathrm{~W}$ at $80 \mathrm{~K}$.

The CPMUs offer further advantages over SCUs, with the saving of electricity and a stable operation without any quench. In addition, all techniques of magnetic field correction developed for permanent magnet undulators can be applied to the CPMUs without any significant modification.

\section{CHARACTERISTICS OF NdFeB MAGNETS AT CRYOGENIC TEMPERATURES}

Sintered NdFeB magnets exhibit negative dependence of remanent fields against temperature, typically $-0.1 \% / \mathrm{K}$ around room temperature. According to this

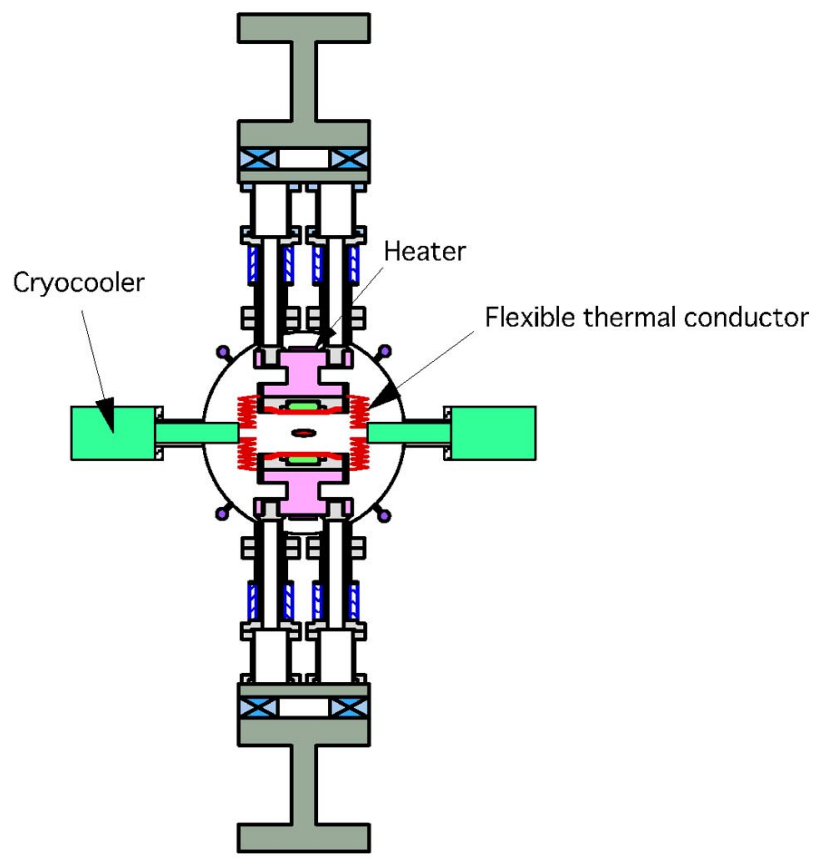

(b)

FIG. 1. (Color) Design examples of a CPMU with refrigerant channels (a) or with cryocoolers (b). 
constant, the magnetic field increases as lowering the magnet temperature. Nevertheless there is a turning temperature around $140 \mathrm{~K}$, below which the magnetic field starts to decrease due to spin reorientation. The spin reorientation refers to a change in the preferred direction of the magnetization with respect to the easy axis of magnetization [13].

In order to find the optimum temperature, the remanent fields of commercially available $\mathrm{NeFeB}$ magnets were measured. The magnetic fields were measured using a calibrated hall probe attached to the surface of a rectangular sample magnet $(46 \mathrm{~mm} \times 12 \mathrm{~mm} \times 8 \mathrm{~mm})$. The sample magnets are magnetized at room temperature along the axis of $12 \mathrm{~mm}$. The measured fields at the position of the hall probe were converted to the remanent fields $\left(B_{r}\right)$ assuming permeance $P=1.77$ and recoil permeability $\mu_{r}=1.05$.

Figure 2 shows the measured remanent fields of the NdFeB and PrFeB magnets, NEOMAX 35EH, 50BH, and 53CR (Sumitomo Special Metal Co., Ltd.), as a function of temperature. NEOMAX 35EH, which has high coercivity but a medium remanent field, is used for conventional in-vacuum undulators in SPring-8. NEOMAX $50 \mathrm{BH}$ is a typical magnet with a high remanent field but low coercivity at room temperature. The optimum temperatures for $35 \mathrm{EH}$ and $50 \mathrm{BH}$ are slightly different, but both magnets show similar behavior: the magnetic field decreases below a certain temperature. In contrast, the remanent field of 53CR, which is a $\mathrm{PrFeB}$ magnet containing praseodymium (Pr) in substitution for neodymium $(\mathrm{Nd})$, continues to increase at low temperatures as shown in Fig. 2. It should be mentioned that the change of the magnetic fields in Fig. 2 is entirely reversible with respect to the temperature.

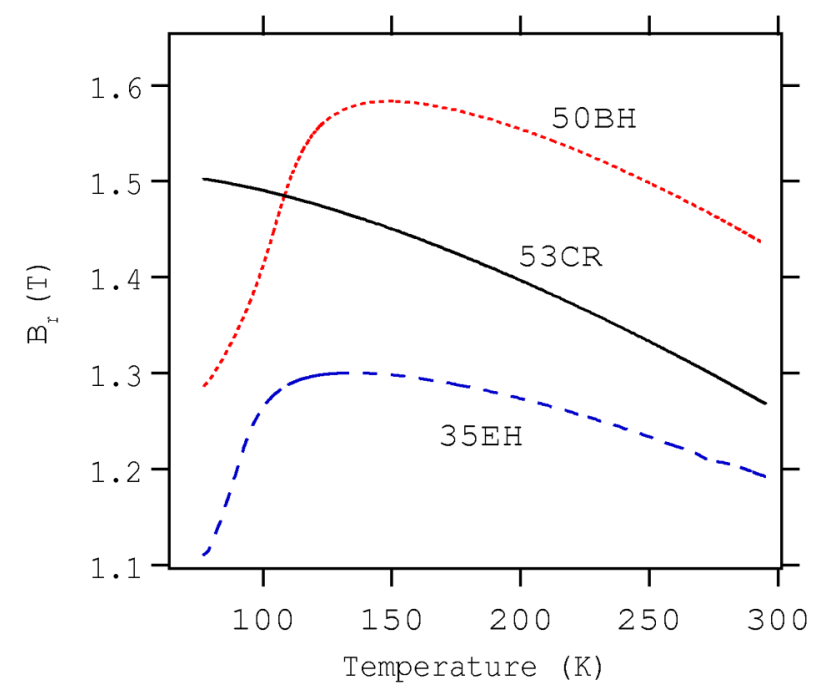

FIG. 2. (Color) Temperature dependence of the remanent fields $\left(B_{r}\right)$ of sintered $\mathrm{NdFeB}$ magnets $(35 \mathrm{EH}$ and $50 \mathrm{BH})$ and a PrFeB magnet (53CR).
Meanwhile, the coercivity of these three magnets significantly increases at low temperatures. The coercivity was measured with a superconductive magnetometer (Quantum Design MPMS XL7), which can supply $\pm 7 \mathrm{~T}$ to a temperature controlled sample. Figure 3 is the measured coercivity $\left({ }_{i} H_{c}\right)$ of $35 \mathrm{EH}, 50 \mathrm{BH}$, and $53 \mathrm{CR}$ magnets as a function of temperature.

Recent work reveals that the resistance of magnetization against electron beam irradiation relates to coercivity in the case of $\mathrm{NdFeB}$ magnets [14]. For example, a $\mathrm{NdFeB}$ magnet with high coercivity (NEOMAX 27VH) has the same resistance as $\mathrm{Sm}_{2} \mathrm{Co}_{17}$ magnets [14], which were believed to be the most resistant against electron irradiation among rare earth magnets [15]. The coercivity of $27 \mathrm{VH}$ is about $\mu_{0}{ }_{i} H_{c}=3.6 \mathrm{~T}$ at room temperature. Comparing this value to those in Fig. 3, even the coercivity of 50BH, which is the lowest among the measured three magnets, exceeds $3.6 \mathrm{~T}$ below $150 \mathrm{~K}$. From these results, the magnets of CPMUs are expected to have not only better magnetic field performance, but also better resistance to electron beam irradiation than the room temperature in-vacuum devices. The improvement of radiation resistance at low temperatures was already confirmed for $\mathrm{NdFeB}$ magnets using proton beams [16].

\section{EXPECTED PERFORMANCE OF CPMUS}

In order to estimate the performance of CPMUs, three undulator types with a periodic length of $14 \mathrm{~mm}$ are considered. As a pure permanent magnet undulator, $50 \mathrm{BH}$ is supposed to be used at $148 \mathrm{~K}\left(B_{r}=1.58 \mathrm{~T}\right)$ in a Halbach configuration (magnet size: $35 \mathrm{~mm}$ in width $\times$ $10 \mathrm{~mm}$ in height). The other two undulators are hybrid undulators. The first one uses $50 \mathrm{BH}$ as magnets and permendur as a pole material. The other hybrid undulator

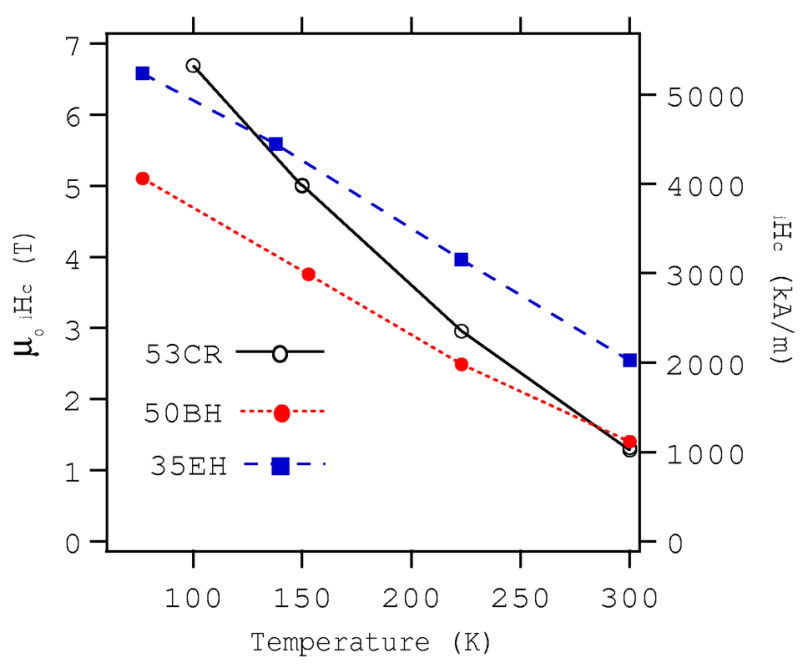

FIG. 3. (Color) Temperature dependence of the coercivity $\left({ }_{i} H_{c}\right)$ of sintered $\mathrm{NdFeB}$ magnets $(35 \mathrm{EH}$ and $50 \mathrm{BH})$ and a PrFeB magnet (53CR). 
uses a combination of 53CR magnets and polycrystalline dysprosium (Dy) poles at $77 \mathrm{~K}\left(B_{r}=1.5 \mathrm{~T}\right)$. Dysprosium is known for its high saturation field $(3.2 \mathrm{~T}$ at $77 \mathrm{~K})$ at cryogenic temperatures below $88 \mathrm{~K}$ [17]. In both hybrid undulators, additional magnets are placed beside poles in order to increase the magnetic field as shown in Fig. 4 [18]. The thickness of poles is $2.5 \mathrm{~mm}\left(0.178 \lambda_{u}\right)$ for the 50BH hybrid undulator and $2.1 \mathrm{~mm}\left(0.15 \lambda_{u}\right)$ for the $53 \mathrm{CR}$ hybrid undulator, respectively, where $\lambda_{u}$ is an undulator periodic length. Figure 5 compares the peak fields of three $14 \mathrm{~mm}$ period undulators as a function of the magnetic gap calculated using RADIA [19]. As a reference, a plot for a room temperature pure magnet undulator made with $35 \mathrm{EH}$ magnets $\left(B_{r}=1.19 \mathrm{~T}\right)$ is also added in the figure.

In fact, the deflection parameter $K$ of an undulator is more important than the peak field, since it is $K$ that determines the energy of radiated photons as

$$
\lambda=\frac{\lambda_{u}}{2 \gamma^{2}}\left(1+\frac{K^{2}}{2}\right)
$$

where $\lambda$ is a photon wavelength and $\gamma$ is a Lorentz factor of electron beams. $K$ is effectively defined by

$$
\begin{gathered}
K^{2}=2\left(\frac{e}{m_{e} c}\right)^{2}\left\langle\left(I_{y}-\left\langle I_{y}\right\rangle\right)^{2}\right\rangle, \\
I_{y}(z)=\int_{-\lambda_{u} / 2}^{z} B_{y}\left(z^{\prime}\right) d z^{\prime},
\end{gathered}
$$

where $m_{e}$ and $e$ are the electron mass and charge, $B_{y}(z)$ is a magnetic field, and $c$ is the speed of light [20,21]. \langle\rangle indicates an average over one undulator period.

Figure 6 shows the attainable $K$ of the three CPMUs in terms of the undulator period at the gaps of 3 and $5 \mathrm{~mm}$. Comparing with the room temperature in-vacuum undulator, the performance of the magnetic fields is improved by roughly $30 \%$ for the CPMU of a pure magnet type and by $50 \%$ for the CPMUs of hybrid types.

Table I is a comparison of the magnetic gaps among the CPMUs, the latest SCU developed by ACCEL Instruments $\mathrm{GmbH}$ [7] and the room temperature invacuum undulator under common conditions of $K=1.8$ and $\lambda_{u}=14 \mathrm{~mm}$. In case of the room temperature invacuum undulator, the magnetic gap should be closed to $1.9 \mathrm{~mm}$ to fulfill the conditions. However, the gap is eased

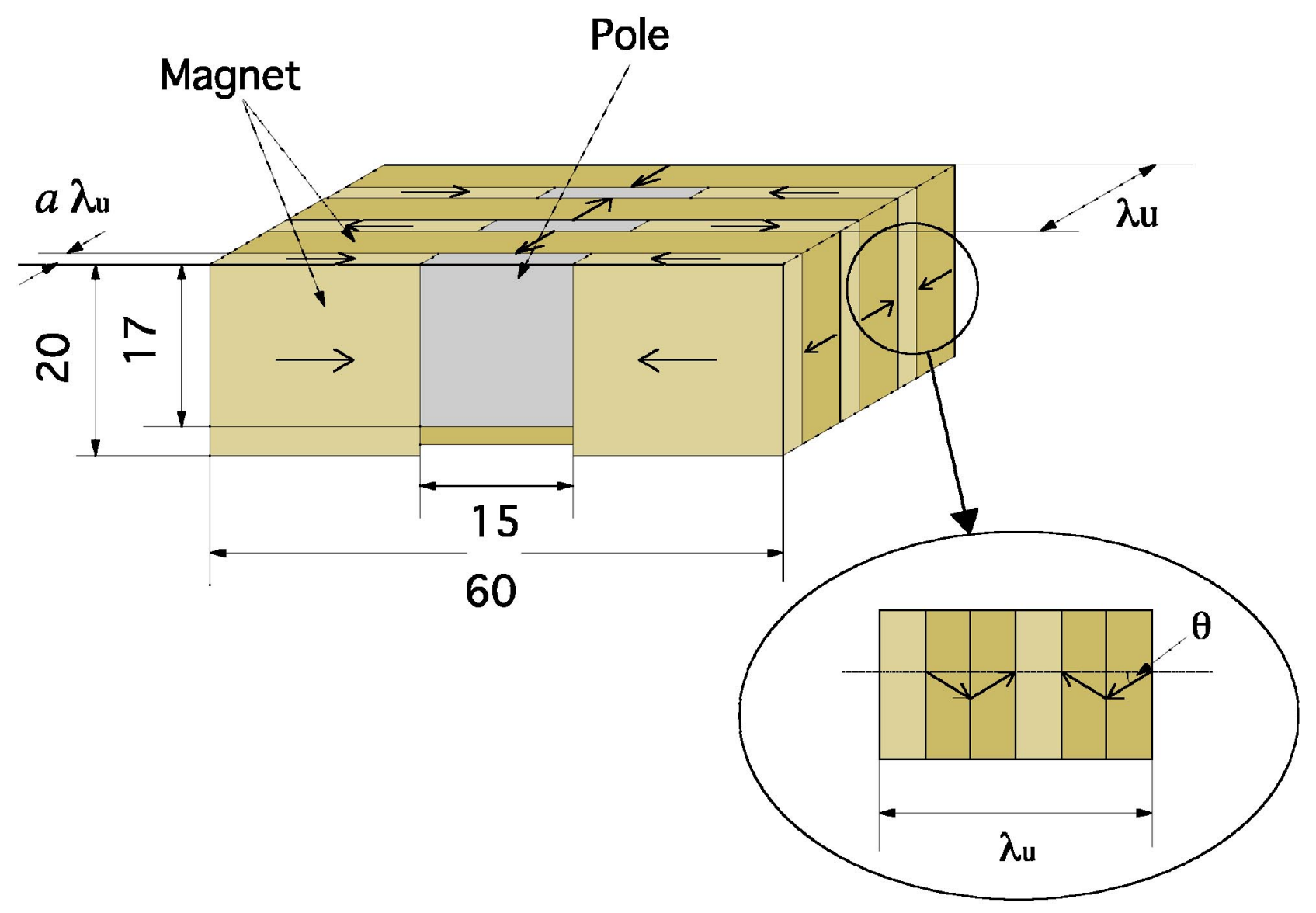

FIG. 4. (Color) Dimensions (mm) of hybrid CPMU used for calculations. $a \times \lambda_{u}$ is a thickness of poles, where $a$ is 0.178 for the $50 \mathrm{BH}$ undulator and 0.15 for the 53CR undulator. $\lambda_{u}$ is an undulator periodic length. Permendur and dysprosium are used as pole materials for the $50 \mathrm{BH}$ and $53 \mathrm{CR}$ undulators, respectively. The case of tilted magnetization by an angle $\theta$ is shown in the circle. 


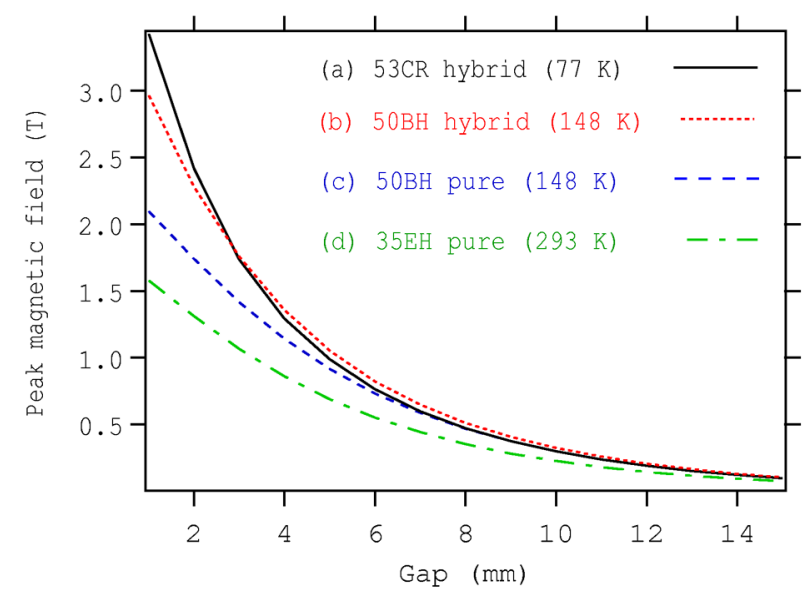

FIG. 5. (Color) Peak magnetic field calculated for $14 \mathrm{~mm}$ period undulators. (a) 53CR + Dy hybrid CPMU (77 K); (b) $50 \mathrm{BH}+$ permendur hybrid CPMU $(148 \mathrm{~K})$; (c) $50 \mathrm{BH}$ pure magnet CPMU $(148 \mathrm{~K})$; (d) conventional 35EH pure magnet undulator (room temperature).

to be $3.2 \mathrm{~mm}$ for the CPMU of a pure magnet type. This gap value is practically feasible for a storage ring operation, if we come to think of the in-vacuum undulator being operated at a $3.3 \mathrm{~mm}$ gap in NSLS [2]. The necessary gap is further enlarged to $3.8 \mathrm{~mm}$ for the hybrid CPMU.

Further improvement of the magnetic performance is still possible for the CPMUs, for example, by optimizing the direction of magnetization. If the direction of magnetization is optimized with respect to the angle $\theta$ in Fig. 4, the electron beam trajectory becomes closer to sinusoidal and $K$ slightly increases. As shown in the last column of Table I, the gap can be increased to $4.0 \mathrm{~mm}$ for a hybrid CPMU with $\theta=20^{\circ}$.

\section{DISCUSSION}

In order to make the physical aperture for electron beams the same as the magnetic gap in SCUs, superconductive magnets should be exposed to the electron beams [22]. Considering the capacity of current crycoolers at the temperature of liquid helium, the heat load due to the resistive wall effect and synchrotron

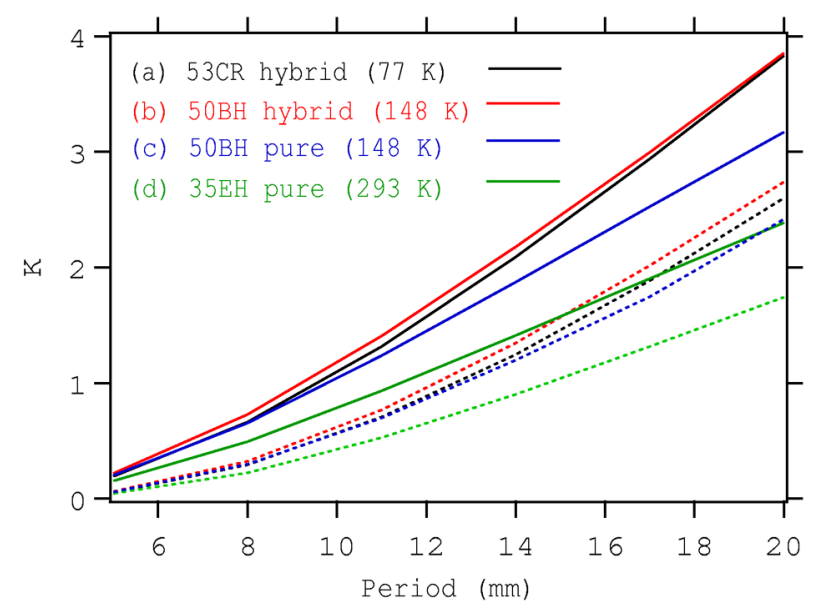

FIG. 6. (Color) $K$ parameters of four different undulators at the gaps of $3 \mathrm{~mm}$ (solid lines) and $5 \mathrm{~mm}$ (dotted lines). (a) 53CR + Dy hybrid CPMU (77 K); (b) $50 \mathrm{BH}+$ permendur hybrid CPMU (148 K); (c) 50BH pure magnet CPMU (148 K); (d) conventional 35EH pure magnet undulator (room temperature). $K$ parameters are calculated according to Eq. (2).

radiation should be smaller than a few watts to prevent quench. Otherwise thermal shields should be inserted between the electron beam and the superconductive magnets at the expense of an effective gap loss. In the case of Table I, if the thickness of the shields is more than $3 \mathrm{~mm}$, the performance of SCUs becomes practically the same as that of conventional in-vacuum undulators. On the other hand, the magnetic gap of the CPMUs corresponds to the physical aperture for the electron beams except an additional $0.1 \mathrm{~mm}$ gap loss due to the metal sheets covering the magnet surface [12].

In the CPMUs, the thermal budget problem is not so serious because compact cryocoolers with high cooling capacity are available at cryogenic temperatures higher than the liquid nitrogen temperature. The reliability of the CPMU operation is not degraded when compared to that of conventional in-vacuum undulators, since there is no possibility for quench. Although field measurements may have to be carried out at cryogenic temperatures, the field correction techniques developed for conventional permanent magnet undulators can be directly applied to

TABLE I. Comparison of magnetic gaps among the CPMUs, the SCU developed by ACCEL [7], and a room temperature invacuum undulator under common conditions of $K=1.8$ and $\lambda_{u}=14 \mathrm{~mm} . K$ parameters of the in-vacuum undulator and CPMUs are calculated according to Eq. (2). The magnet material of the CPMUs is 50BH operating at $148 \mathrm{~K}$.

\begin{tabular}{lccccc}
\hline \hline & $\begin{array}{c}\text { ACCEL } \\
\text { SCU } \\
{[7]}\end{array}$ & $\begin{array}{c}\text { Room temperature } \\
\text { pure magnet } \\
\left(B_{r}=1.19 \mathrm{~T}\right)\end{array}$ & $\begin{array}{c}\text { CPMU } \\
\text { pure magnet } \\
\left(B_{r}=1.58 \mathrm{~T}\right)\end{array}$ & $\begin{array}{c}\text { CPMU } \\
\text { hybrid } \\
\left(B_{r}=1.58 \mathrm{~T}\right)\end{array}$ & $\begin{array}{c}\text { CPMU hybrid, } \\
\text { magnetization tilted by 20 } \\
\left(B_{r}=1.58 \mathrm{~T}\right)\end{array}$ \\
\hline $\mathrm{K}$ & & & 1.8 & & \\
Peak magnetic field $(\mathrm{T})$ & 1.3 & 1.35 & 1.37 & 1.43 & 1.39 \\
Magnetic gap (mm) & 5 & 1.9 & 3.2 & 3.8 & 4.0 \\
$1 \%$ field uniformity & $>10 \mathrm{~mm}$ & $>10 \mathrm{~mm}$ & $>10 \mathrm{~mm}$ & $\pm 5 \mathrm{~mm}$ & $\pm 5 \mathrm{~mm}$ \\
\hline \hline
\end{tabular}


the CPMUs. Thus the development of the CPMUs is straightforward from the current in-vacuum undulators and there is no big technological obstruction.

In this paper, we showed the achievable performance of the CPMUs using the currently available technologies of permanent magnets. However, the performance of $\mathrm{NdFeB}$ magnets is still being improved. Particularly, PrFeB magnets have the potential for higher magnetic fields, since they have been almost abandoned for more than ten years because of a lack of demand. If the experience of optimization procedures accumulated on $\mathrm{NdFeB}$ magnets is applied to the manufacture of $\mathrm{PrFeB}$ magnets, further improvement of the CPMU performance can be expected.

\section{ACKNOWLEDGMENTS}

The authors thank Dr. Katsumata and Ms. Kikkawa for the coercivity measurements.

[1] T. Schmidt, G. Ingold, A. Imhof, B. Patterson, L. Patthey, C. Quitmann, C. Schulze-Briese, and R. Abela, Nucl. Instrum. Methods Phys. Res., Sect. A 467-468, 126 (2001).

[2] P. Stefan, T. Tanabe, S. Krinsky, G. Rakowsky, L. Solomon, and H. Kitamura, J. Synchrotron Radiat. 5, 417 (1998).

[3] T. Shintake et al., Nucl. Instrum. Methods Phys. Res., Sect. A 507, 382 (2003).

[4] S. Yamamoto, T. Shioya, M. Hara, H. Kitamura, X. Zhang, T. Mochizuki, H. Sugiyama, and M. Ando, Rev. Sci. Instrum. 63, 400 (1992).

[5] H. Kitamura, J. Synchrotron Radiat. 5, 184 (1998).

[6] G. Ingold, I. Ben-Zvi, L. Solomon, and M. Woodle, Nucl. Instrum. Methods Phys. Res., Sect. A 375, 451 (1996).

[7] S. Kubsky, D. Dölling, A. Geisler, A. Hobl, U. Klein, D. Krischel, H. Moser, R. Rossmanith, and S. Chouhan, in Proceedings of the 8th International Conference on Synchrotron Radiation Instrumentation, San Francisco, 2003 (to be published).
[8] S. Chouhan, R. Rossmanith, S. Strohmer, D. Doelling, A. Geisler, A. Hobl, and S. Kubsky, in Proceedings of the PAC 2003, Portland, OR (IEEE, Piscataway, NJ, 2003).

[9] R. Rossmanith, H. Moser, A. Geisler, A. Hobl, D. Krischel, and M. Schillo, in Proceedings of the EPAC 2002, Paris, France (EPS-IGA/CERN, Geneva, 2002), p. 2628.

[10] E. Wallén, in Proceedings of the Workshop on Superconducting Undulators and Wigglers, Grenoble, France, 2003 (ESRF, Grenoble, 2003).

[11] M. Sagawa, S. Hirosawa, Y. Otani, H. Miyajima, and S. Chikazumi, J. Magn. Magn. Mater. 70, 316 (1987).

[12] T. Hara, T. Tanaka, T. Tanabe, X. Maréchal, S. Okada, and H. Kitamura, J. Synchrotron Radiat. 5, 403 (1998).

[13] L. García, J. Chaboy, F. Bartolomé, and J. Goedkoop, Phys. Rev. Lett. 85, 429 (2000).

[14] T. Bizen, Y. Asano, T. Hara, X. Maréchal, T. Seike, T. Tanaka, H. Kitamura, H. Lee, and D. Kim, in Proceedings of the 8th International Conference on Synchrotron Radiation Instrumentation, San Francisco, 2003 (Ref. [7]).

[15] X. Maréchal and T. Shintake, in Proceedings of the 8th International Conference on Synchrotron Radiation Instrumentation, San Francisco, 2003 (Ref. [7]).

[16] O.-P. Kähkönen, S. Mäkinen, M. Talvitie, H. Rajainmäki, and M. Manninen, Europhys. Lett. 12, 413 (1990).

[17] W. Swift and M. Mathur, IEEE Trans. Magn. 10, 308 (1974).

[18] T. Shioya, S. Yamamoto, S. Sasaki, M. Katoh, Y. Kamiya, and H. Kitamura, Rev. Sci. Instrum. 60, 1855 (1989).

[19] O. Chubar, P. Elleaume, and J. Chavanne, J. Synchrotron Radiat. 5, 481 (1998).

[20] When the magnetic field is sinusoidal, $K$ can be expressed using the peak field $B_{p}$ as $K=$ $\left[\left(e B_{p} \lambda_{u}\right) /\left(2 \pi m_{e} c\right)\right]$.

[21] T. Tanaka, K. Shirasawa, and H. Kitamura, Rev. Sci. Instrum. 73, 1724 (2002).

[22] S. Prestemon, D. Dietderich, S. Marks, and R. Schlueter, in Proceedings of the 8th International Conference on Synchrotron Radiation Instrumentation, San Francisco, 2003 (Ref. [7]). 